\title{
Análisis y evaluación de la pesca deportiva como atractivo turístico en el noroeste de Santa Cruz
}

\section{Analysis and evaluation of fly fishing as tourist attraction in northwest of Santa Cruz}

\author{
Carlos Javier Andrade, Juan Manuel Biott, Silvia Ferrari \\ carlos.andrade.94@hotmail.com \\ Universidad Nacional de la Patagonia Austral, Unidad Académica Río Gallegos. Domicilio: \\ Avda. Gregores y Piloto Lero Rivero s/n. Río Gallegos (Santa Cruz, Argentina)
}

Recibido: 28/05/2020. Aceptado: 14/12/2020

\section{RESUMEN}

La Ruta Nacional $\mathrm{N}^{\circ} 40$ es uno de los corredores turísticos más promocionados del país. Atraviesa el país de sur a norte, y el kilómetro 0 se encuentra en la provincia de Santa Cruz (Argentina), la cual recorre uniendo localidades con importantes atractivos turísticos y recursos potenciales que propician la práctica de diversas actividades turísticas, tradicionales y alternativas. La pesca deportiva, englobada dentro del turismo de intereses especiales (TIE), es una de las actividades más relevantes a nivel internacional, y la provincia de Santa Cruz cuenta con destinos consolidados y otros propicios para su desarrollo. A pesar del gran potencial de la actividad, su crecimiento a nivel mundial y su visible aporte hacia un desarrollo sustentable en la zona noroeste de la provincia, aledaña a la RN40, no se encuentran diagnósticos ni inventarios sobre lugares donde se desarrolle o podría efectuarse la actividad; lo cual es necesario para su mejor gestión y promoción.

El trabajo, realizado en el marco de una Beca CIN para estudiantes, ha iniciado en junio del presente año y propone inventariar, jerarquizar y cartografiar los lugares de pesca, así como conocer su estado actual y limitaciones para mejorar la toma de decisiones y optimizar su aprovechamiento turístico en dicho territorio. Se presentan los resultados de la primera etapa del estudio, abordando aquellos lugares de pesca que están siendo explotados turísticamente en la actualidad. Para ello, se trabajó mediante entrevistas a informantes claves, privados y públicos, y mediante la recolección de aquella información que contribuya a una planificación turística integral, enmarcada dentro del desarrollo sustentable.

Palabras clave: Turismo de intereses especiales; pesca deportiva; desarrollo sustentable.

\begin{abstract}
The route $\mathrm{N}^{\circ} 40$ is one of the most promoted routes in the country. It crosses the country from the south to the north, and kilometer 0 is in Santa Cruz. This route joins locations that have important tourist attractions and potential resources that are suitable for practicing different tourist activities, traditional and alternatives. Sport fishing is included in special interest tourism. It is one of the most relevant activities internationally. Santa Cruz has consolidated destinations and other potentials to its development. Although the importance of the activity, its growth and its contribution to sustainable development, in the northwest of Santa Cruz, there are no diagnoses or inventories on places where sport fishing is practiced or where it could be practiced. All this is necessary for better management and promotion.
\end{abstract}


The report, made through a scholarship CIN, proposes to inventory the fishing areas in the northwest inquire about their current status and limitations to improve decision making and optimize its tourism use in study area. The results of the first stage of the study are presented, addressing those fishing site that are currently being exploited tourism. To do this, it's done through interviews with key informants, private and public, and by collecting information that contributes to a comprehensive tourism planning, framed within sustainable development.

Keywords: Special interest tourism; sport fishing; sustainable development.

\section{INTRODUCCIÓN}

El turismo representa una de las principales alternativas de desarrollo socio-económico y cultural para Latinoamérica, contribuyendo no sólo como fuente de divisas para las economías nacionales, sino además beneficiando directamente a otros sectores de la población, revitalizando las cadenas de valor y el empoderamiento productivo de las empresas proveedoras de bienes y servicios (Vacca et al., 2008). La Patagonia ofrece multiplicidad de recursos y atractivos turísticos, algunos ya consolidados y otros potenciales que requieren de su puesta en valor y una planificación ordenada, teniendo en cuenta las necesidades del visitante, las particularidades y el estado de conservación del recurso (Ferrari et al., 2015; Mazzoni, 2017).

En Santa Cruz, uno de los corredores turísticos particularmente promocionados es la Ruta Nacional 40, la cual tiene aquí su kilómetro cero; en tanto que su finalización es el kilómetro 5.244 en Jujuy, siendo la más extensa y espectacular de Argentina (http://www.ruta40.gov.ar). Recorre $1.401 \mathrm{~km}$ en toda la provincia, uniendo distintos puntos turísticos con variados recursos naturales/culturales, que propician el desarrollo de modalidades turísticas alternativas, como la denominada "Turismo de Intereses Especiales" (TIE). La pesca deportiva es una de las actividades de TIE más relevantes a nivel internacional. Los pescadores están dispuestos a viajar grandes distancias en busca de regiones con atributos excepcionales para practicar esta actividad (Soto et al., 2001; U.S Fish and Wildlife Service, 2016; Molina García, 2013). Tal es el caso de la pesca deportiva de salmónidos (trucha arco iris, marrón, fontinalis, salmones del Pacífico y plateado), que se realiza en diferentes ambientes acuáticos de Santa Cruz, como en los ríos Gallegos y Santa Cruz (Pascual et al., 2001), que ya poseen renombre mundial. Es una de las actividades turísticas de mayor crecimiento y potencialidad en la provincia, ya que cuenta con recursos pesqueros que resultan atractivos no sólo para pescadores nacionales sino también para un número importante de pescadores extranjeros, quienes incurren en altos costos de viaje y estadía en busca de un producto turístico de muy alta calidad (Biott, 2016).

Sin embargo, algunos pesqueros con importante potencial turístico, como los ubicados en el noroeste de Santa Cruz y aledaños a la RN40, no se encuentran plenamente desarrollados ni cuentan con diagnósticos sistematizados y/o inventarios, de modo que permitan planificar un crecimiento ordenado de la actividad. Esta situación conlleva a una escasa previsibilidad para atender a las demandas de los pescadores, como ser la accesibilidad a los sitios de pesca, existencia de infraestructuras y servicios adecuados o el conocimiento de las especies de su valor pesquero, entre otras (Navarro et al., 2014).

Los modelos actuales de planificación del desarrollo turístico, conciben el territorio como un producto integral que suma paisaje, empresas, infraestructuras, equipamientos, entorno social y patrimonio (Calderón Vázquez, 2010). En este contexto, es necesaria una identificación de los recursos con potencial turístico en el territorio y su distribución espacial, el reconocimiento del estado actual y del entorno, así como su jerarquización en función a 
diferentes variables que permitan la comparación entre diferentes ofertas, como una de las condiciones inherentes de una planificación turística integral. También es importante conocer el perfil de los pescadores que mayormente visitan la provincia, ya que los intereses y requerimientos pueden diferir entre ellos, lo cual permitiría diseñar a futuro campañas de marketing personalizadas y que potencien las aptitudes que ofrece la zona.

El presente trabajo, realizado en el marco de una Beca de Estímulo a las Vocaciones Científicas-CIN para estudiantes, se ha iniciado en junio del presente año y propone inventariar, jerarquizar y cartografiar los lugares de pesca, actuales y potenciales, así como conocer su estado actual y limitaciones para mejorar la toma de decisiones y optimizar su aprovechamiento turístico en dicho territorio, enmarcado dentro del desarrollo sustentable. Se presentan los resultados de la primera etapa del estudio, abordando aquellos lugares de pesca que están siendo explotados, en la actualidad.

\section{MARCO TEÓRICO}

La pesca deportiva se define como la pesca de diferentes especies de agua salada o dulce, con artes y métodos considerados no perjudiciales para la conservación de la fauna ictícola, y cuyo fin sea el disfrute sin comerciar las especies capturadas. (Decreto $\mathrm{N}^{\circ}$ 3772/07 Reglamentación de Turismo Aventura de Santa Cruz).

La pesca con mosca (conocido internacionalmente en inglés como "fly fishing") es la modalidad más famosa. Cada vez genera más adeptos a practicar y utilizar las técnicas de tiro que precisa dicha modalidad. Para este tipo de pesca se utiliza un señuelo artificial diseñado mayormente utilizado plumas, al que se denomina mosca por su forma, creada para representar un insecto o un pez pequeño (SENATUR, 2001).

Además de la devolución al hábitat de las especies capturadas, la pesca deportiva necesita de un ambiente natural de calidad paisajística y en condiciones para generar una experiencia satisfactoria. El fin de esta tipología de pesca no es alimenticio, es esparcimiento y satisfacción personal, que contribuye a la liberación de estrés (Cajade, 2009).

La pesca deportiva está englobada dentro de lo que se conoce como turismo alternativo, y se la puede reconocer como una de las actividades más relevantes a nivel internacional de la tipología conocida como Turismo de Intereses Especiales (TIE). La Organización Mundial de Turismo reconoce al TIE como una amplia categoría de turismo basado en intereses concretos que incluye naturaleza, cultura, historia u otros campos de índole diversa ofrecidos en un entorno local (OMT, 2008). Este tipo de turismo se refiere a formas distintas de viajes, con motivaciones más específicas que las vacaciones tradicionales o habituales. Se caracteriza por no ser masivo, entregar garantías respecto al cuidado del ambiente y, además, por focalizar la importancia en la experiencia (Robin et al., 2015).

Los pescadores están dispuestos a viajar grandes distancias en busca de regiones con atributos excepcionales para practicar la actividad. A la hora de decidir los sitios a los que se desplazarán, tienen en cuenta variables como: la facilidad de captura, las tallas de los peces y la presencia de otros pescadores (Parrado y Huaquin, 2010).

El interés de los pescadores se concentra casi de manera exclusiva en pescar, es decir, que no dirigen su atención hacia atractivos o actividades distintas. Es una actividad capaz de motivar largos desplazamientos para vivir la experiencia de pescar una especie en particular o en ambientes difíciles de encontrar en otros sitios. Los sitios analizados consisten en lugares aislados, difíciles de acceder y donde todo está concentrado en la pesca, que responden a las necesidades de los turistas.

La pesca fly fishing no representa un número significativo dentro de las explotaciones basadas en las capturas de peces, pero se encuentra en un crecimiento constante adquiriendo nuevos 
adeptos año a año (U.S Fish and Wildlife Service, 2016). Según la ONG estadounidense Outdoor Foundation, la pesca deportiva con mosca es una de las tipologías menos populares, representando un 2,3\% total de la pesca en general del país norteamericano. Sin embargo, se encuentra en un crecimiento constante desde el año 2012 en adelante, estimándose un alza de 1 millón de nuevos pescadores, llegando así a la cifra de 7 millones de personas que practican la modalidad (Outdoor Foundation, 2018).

En Argentina, no se encuentran estadísticas reales o actualizadas sobre la actividad, siendo uno de los déficits de esta tipología de turismo. En 2012, datos presentados en el I Congreso de Pesca Deportiva y Turismo, estimaban que nuestro país contaba con un mercado local de 5 millones de pescadores deportivos aproximadamente, concentrándose $40 \%$ en Capital Federal y Provincia de Buenos Aires (Biott, 2012).

El turismo de intereses especiales responde en líneas generales a las premisas planteadas por el turismo sustentable, entendiéndose, según la OMT (1995) como "aquel que tiene plenamente en cuenta las repercusiones actuales y futuras, económicas, sociales $y$ medioambientales para satisfacer las necesidades de los visitantes, la industria, del entorno y de las comunidades anfitrionas".

La pesca deportiva es capaz de despertar un interés en la conservación de los ambientes, ya que la actividad depende exclusivamente del buen estado de los ambientes acuáticos, además que es una actividad dinamizadora dentro de las economías regionales y locales. Posee un enorme potencial económico, al generar flujos de empleos, divisas y expandir sus efectos a otras actividades y servicios (Ibáñez, 2011). Por ejemplo, en países como Costa Rica los ingresos generados por la pesca deportiva sobrepasan los ingresos de la pesca comercial (Universidad de Costa Rica, 2010). Por esta razón, es que comienza a visibilizarse como una de las tipologías turísticas con mayor potencial para llevarse adelante bajo las premisas del turismo sustentable. En este contexto, se desarrolla el presente trabajo, que cuenta entre sus objetivos realizar un diagnóstico integral y caracterización de la pesca deportiva como producto turístico en el noroeste de Santa Cruz, a fin de aportar bases para su planificación ordenada y sustentable.

\section{METODOLOGIA}

El área de estudio abarca el espacio geográfico ubicado entre las localidades de Gobernador Gregores y Perito Moreno, de la provincia de Santa Cruz (Patagonia Austral, Argentina), siguiendo la traza del corredor turístico RN40, en particular sobre los cuerpos de agua próximos, que son base de la pesca deportiva en la actualidad para dicha actividad (Figura 1).

A los efectos de proceder a la valoración de los recursos ícticos y lugares de pesca, se aplicó la metodología de "Inventario y Jerarquización de recursos y atractivos turísticos", siguiendo las propuestas de OEA-Cicatur (1978) y Mincetur (2006), sumado a criterios de catalogación y evaluación acorde a las características de la región, los que fueron adaptados por el equipo de trabajo en el que se inscribe la presente propuesta (Navarro et al., 2014; Mazzoni y Ferrari, 2014; Ferrari et al., 2015).

En el cierre de la temporada 2018-2019, se realizó un recorrido de campo en el lodge "Eagle Valley", administrado por la empresa "Tres Amigos", que se encuentra a orillas del Lago Olnie, lo que permitió conocer in situ un destino de pesca deportiva y efectuar una entrevista semiabierta al encargado del lodge, así como dialogar con los guías de pesca que trabajaban en el sitio. Asimismo, se indagó a los turistas que se encontraban en el lugar, con el fín de

\footnotetext{
1 Alojamiento ubicado en cercanías a las zonas de pesca, con equipamiento y servicios destinados exclusivamente a los pescadores.
} 
recabar información sobre su procedencia, y principalmente los intereses y motivaciones que los llevan a desplazarse a sitios como los que se analizarán en este trabajo.

En primera instancia, se completaron fichas de inventario de los tres lugares de pesca administrados y manejados por empresas privadas, con información obtenida a partir de entrevistas semi estructuradas a dos (2) informantes clave del lodge "Eagle Valley" y "Jurassic Lake", que opera en lago Strobel, complementándose éstos y los restantes con datos de los sitios web donde los prestadores exhiben sus servicios.

El inventario considera al menos, 10 criterios de valoración (Estado actual de conservación del recurso, Estado actual de conservación del entorno, Particularidad del recurso, Accesibilidad legal (pública o privada), física y temporal, Servicios e Infraestructuras turísticas, Vulnerabilidad, Explotación del recurso y Tipo de demanda). Se entiende por "explotación del recurso" al nivel de desarrollo y consolidación del ambiente como lugar de pesca, mientras que el criterio "tipo de demanda" pretende identificar la procedencia de los turistas (regionales, nacionales, internacionales). A cada uno de estos criterios, se asignó una categoría (muy bueno, bueno, regular y malo) y un valor (del 1 al 6), cuya sumatoria proporcionó el puntaje total de cada recurso analizado. Para las puntuaciones otorgadas, se utilizó la información recopilada previamente (Anexo, Tabla 1).

En una segunda etapa, y siguiendo la metodología citada, se estableció un factor de ponderación para aquellos criterios que se consideraron más sobresalientes, según la información recolectada y los aportes de los informantes clave, y que resultaran decisivos en el momento de valorar positivamente un sitio para la pesca, como: la particularidad del recurso íctico, vulnerabilidad y estado de conservación del recurso. Ello permitió obtener el valor final por cada recurso inventariado y el listado jerarquizado, según cinco niveles o categorías de recursos turísticos. Se trata de un proceso comparativo para establecer un orden de importancia de los recursos turísticos inventariados (Anexo, Tabla 2). El puntaje máximo posible a obtener por cada ambiente analizado es de 78, incluyendo la ponderación de los criterios mencionados anteriormente, por lo cual los porcentajes de valoración final fueron obtenidos en función de este número como el $100 \%$.

Para conocer en profundidad la actividad, su impacto y posibles sitios potenciales de pesca, además de las entrevistas dirigidas hacia actores del sector privado, también se ha realizado una entrevista abierta al encargado de Promoción y Marketing de la Secretaría de Turismo de Santa Cruz, y otra dirigida a un representante de la Dirección de Pesca Continental (dependiente de la Secretaría de Estado de Pesca y Acuicultura), para conocer las perspectivas, funciones y acciones que desarrollan ambas instituciones estatales respecto a la pesca deportiva. Cabe destacar que, en el caso de ésta última, es la autoridad de aplicación, siendo quienes deben controlar que se respeten los reglamentos de pesca continental vigentes.

\section{RESULTADOS}

\section{a) Áreas de pesca deportiva}

Se han identificado tres lugares de pesca que operan en la actualidad en el NO de Santa Cruz: lago Strobel, lago Olnie y lago Quiroga (Figura 1), donde funcionan cinco operadores privados, mediante la instalación de lodges, que además de brindar servicio de alojamiento y contar con equipamiento, tienen en su staff guías de pesca experimentados. Los tres se encuentran a escasa distancia de la promocionada RN40 (Tabla 1). 


\begin{tabular}{|ccc|}
$\begin{array}{c}\text { Nombre del ambiente } \\
\text { acuático }\end{array}$ & $\begin{array}{c}\text { Distancia en km. } \\
\text { respecto a RN40 }\end{array}$ & $\begin{array}{c}\mathbf{N}^{\circ} \text { de lodges en el } \\
\text { área }\end{array}$ \\
\hline Lago Strobel & 23 & 3 \\
\hline Lago Olnie & 56 & 1 \\
\hline Lago Quiroga & 33 & 1 \\
\hline
\end{tabular}

Tabla 1. Distancias de ambientes analizados a la RN40 y cantidad de lodges por lugar

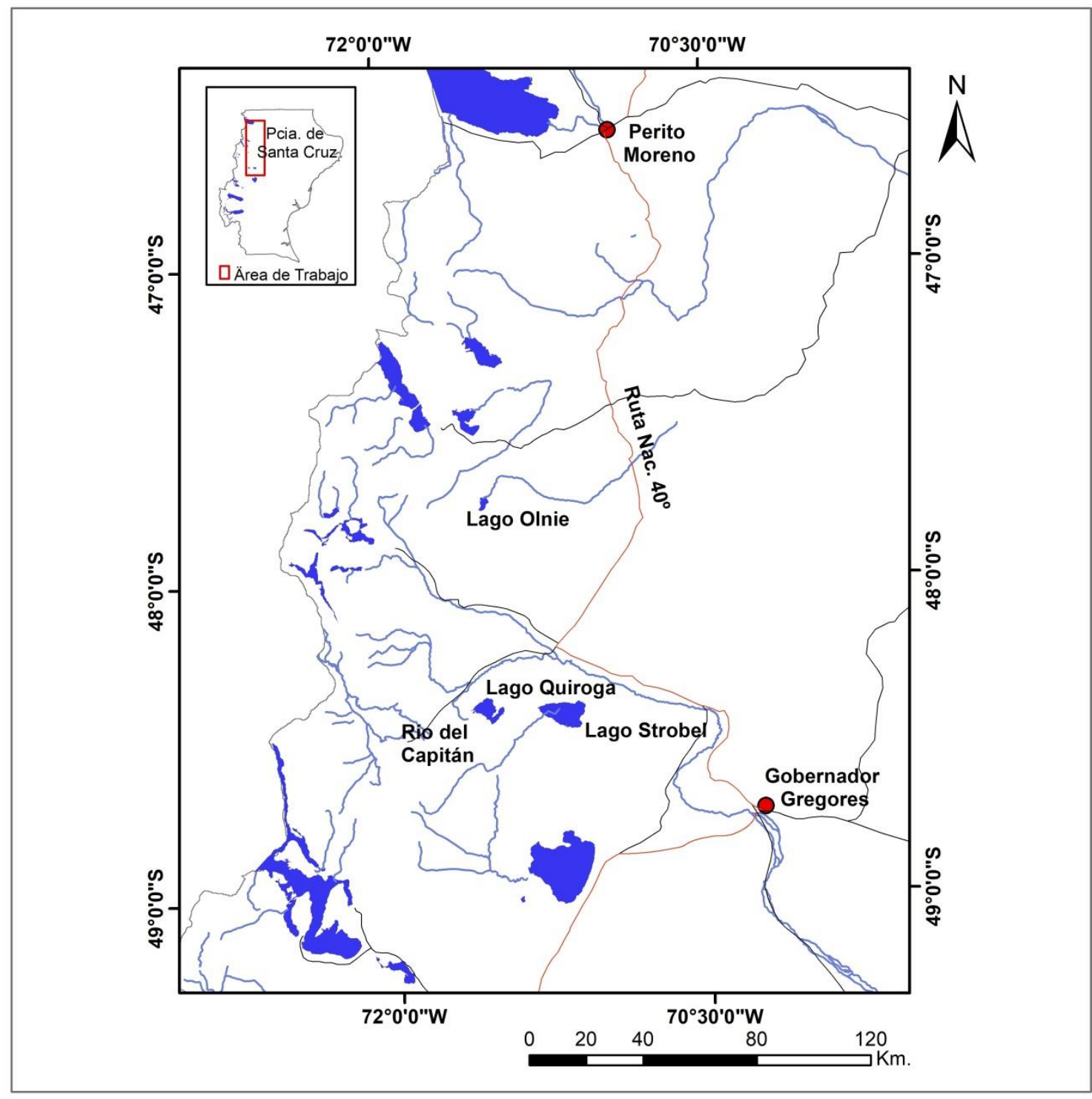

Figura 1. Ubicación del área de estudio en el Noroeste de Santa Cruz y lugares de pesca deportiva analizados. (Fuente: García y Mazzoni, 2000. Modificado por Ing. Daniel Grima, Área de Servicios Técnicos de Cartográfica Teledetección y SIG. UARG-UNPA).

En la siguiente tabla, se presentan los valores de la jerarquización obtenida por cada uno de los tres ambientes analizados, en donde la pesca deportiva se lleva a cabo: 


\begin{tabular}{|lccc|} 
& Lago Olnie & Lago Strobel & $\begin{array}{c}\text { Lago Quiroga/Río } \\
\text { Capitán }\end{array}$ \\
\hline $\begin{array}{l}\text { Estado de } \\
\text { conservación del } \\
\text { recurso }\end{array}$ & 6 & 6 & 6 \\
\hline $\begin{array}{l}\text { Estado de } \\
\text { conservación del } \\
\text { entorno }\end{array}$ & 6 & 6 & 6 \\
\hline Accesibilidad física & 2 & 2 & 2 \\
\hline $\begin{array}{l}\text { Particularidad del } \\
\text { recurso }\end{array}$ & 4 & $\mathbf{5}$ & 4 \\
\hline $\begin{array}{l}\text { Vulnerabilidad } \\
\text { Accesibilidad legal }\end{array}$ & 5 & 5 & 5 \\
\hline $\begin{array}{l}\text { Servicios e } \\
\text { instalaciones }\end{array}$ & 2 & 2 & 2 \\
\hline $\begin{array}{l}\text { Demanda (Tipo de } \\
\text { visitantes) }\end{array}$ & 6 & $\mathbf{5}$ & 6 \\
\hline $\begin{array}{l}\text { Explotación del } \\
\text { recurso }\end{array}$ & 3 & 6 & 5 \\
\hline $\begin{array}{l}\text { VALORACIÓN } \\
\text { TOTAL }\end{array}$ & 38 & $\mathbf{6 3}$ & 40 \\
\hline $\begin{array}{l}\text { VALORACIÓN } \\
\text { PONDERADA }\end{array}$ & 55.5 & $\mathbf{6 2 . 5}$ & \\
\hline $\begin{array}{l}\text { VALOR EN } \\
\text { PORCENTAJE } \\
\text { RESPECTO AL } \\
\text { PUNTAJE } \\
\text { MÁXIMO (78) }\end{array}$ & $\mathbf{7 1 \%}$ & $\mathbf{8 0 \%}$ & $\mathbf{7 2 \%}$ \\
\hline
\end{tabular}

Tabla 2. Jerarquización comparativa de los ambientes acuáticos analizados

Los tres ambientes acuáticos considerados obtuvieron una buena puntuación final, superando el 70\%, con valores similares entre ellos. Lago Strobel destaca con $80 \%$ del puntaje óptimo, mientras que lago Quiroga y lago Olnie poseen un $72 \%$ y un $71 \%$ del máximo respectivamente, los cuales son en todos los casos, considerados de nivel satisfactorio.

La mejor calificación obtenida por el lago Strobel fue debido fundamentalmente, a la talla de las truchas que son posibles de pescar, las cuales alcanzan hasta los 10 kilogramos de peso, motivo por el cual logró el máximo valor dentro del criterio "particularidad del recurso". Asimismo, se destaca por los servicios e instalaciones que ofrece. A diferencia de lago Olnie y lago Quiroga/río Capitán, donde sólo un operador privado ofrece la actividad pesquera, en inmediaciones del lago Strobel funcionan tres lodges de pesca que albergan pescadores y a quienes ofrecen servicios de guía, los que se denominan: "Estancia Laguna Verde", "Estancia Lago Strobel" y "Jurassic Lake". A su vez, la consolidación del destino Strobel para la pesca deportiva, es mucho más alta que las demás, ya que ha conseguido mantener su actividad a lo largo de los años, obteniendo un renombre internacional. Uno de sus lodge, Jurassic Lake, ofrece su servicio desde la temporada 2006/2007.

La actividad turística en lago Olnie, por otra parte, es muy reciente. Su primera temporada de pesca oficial fue la de 2018/2019; mientras que la "Estancia Río Capitán" presta sus servicios para pescadores desde la temporada 2013/2014. 
b) Especie de interés para la pesca deportiva

La especie íctica de interés para la pesca deportiva en los tres ambientes acuáticos, fue la trucha arco iris (Oncorhynchus mykiss), perteneciente a la familia de los salmónidos. Es una especie nativa del hemisferio norte, que fue introducida en distintos ambientes acuáticos de Patagonia por su fácil adaptación e interés deportivo. Por ello, ha tenido una gran expansión, $\mathrm{y}$ en muchos casos ha incidido negativamente sobre la fauna nativa, por su carácter predatorio. Esto ha provocado que organizaciones como la Unión Internacional por la Conservación de la Naturaleza la haya incluido dentro de las 100 especies exóticas invasoras más dañinas del mundo (UICN, 2000).

\section{c) Procedencia y perfil de los pescadores}

Según las respuestas de los informantes clave pertenecientes al sector privado, la mayoría de los turistas son internacionales (más del 90\%). Por esta razón, en las tres fichas de relevamiento, los sitios han obtenido el puntaje más alto posible (6) en el ítem "Tipo de demanda".

Gran parte de los pescadores provienen desde Estados Unidos. El operador del lodge Jurassic Lake, señaló que "podrían cubrir una temporada de trabajo sólo con turistas estadounidenses", lo cual demuestra que la demanda mayormente es de pescadores extranjeros y de dicha procedencia.

Los lodges ofrecen paquetes turísticos de media semana (3 días) o semana completa (6 días más uno de viaje al lugar), y además de los servicios de guía de pesca y alojamiento, se encargan de la logística y traslado de los pescadores.

El pescador que se dirige a este tipo de sitios con difícil acceso, es por lo general un pescador experimentado y con buena posición económica, la mayoría son personas jubiladas y adultos mayores. Según lo mencionado en las entrevistas por los agentes del sector privado, suelen ser "gente que sólo le interesa pescar de a sol a sol y no posee mucho interés en otros tipos de atractivos". Es por ello, que para este tipo de turismo, la conectividad con otros centros turísticos no significa un problema relevante, ya que su interés principal es fundamentalmente la pesca y permanecer en el lugar.

Según señalaron los entrevistados, los turistas aficionados a la pesca deportiva encuentran en Santa Cruz un ambiente ideal para pescar. Lugares aislados, solitarios, incomunicados, de alto valor estético y especialmente, con una calidad de pesca reconocida que convierten a la provincia en uno de los sitios más fuertes y con mayor potencial en esta tipología de turismo, no sólo a nivel regional sino también internacional. "La jerarquía de la calidad de pesca de Santa Cruz es comparable con un atractivo como el Glaciar Perito Moreno o la escalada en Chaltén" fueron palabras de uno de los entrevistados perteneciente al sector privado y encargado de Jurassic Lake. Esta afirmación, a su vez, fue coincidente con la de un representante de la Secretaria de Turismo, al ser consultado en una entrevista realizada en el organismo estatal de la provincia.

\section{d) Limitantes de la pesca deportiva}

La principal limitante de la actividad en los tres lugares fue la accesibilidad, lo cual se reflejó en los bajos puntajes obtenidos en dicho ítem. A excepción del lodge Jurassic Lake, en el lago Strobel, que posee una pequeña pista de aterrizaje, sólo para sus clientes, en los demás no se puede acceder con un vehículo convencional debido a las condiciones de transitabilidad de los caminos. Si bien la RN40 se encuentra pavimentada, los caminos para llegar a los lodges son de ripio, y en días de lluvia se vuelven poco transitables, siendo indispensable contar con vehículos $4 \mathrm{x} 4$. 
La señalización escasa representa otro problema para el acceso a las áreas; lo cual dificulta las logísticas, dedicando proporcionalmente, gran parte del tiempo al traslado a cada lodge, lo que puede traducirse en horas de pesca perdidas.

Los caminos dificultosos y privados a los lugares de pesca tienen como consecuencia que el acceso a las personas que deseen pescar, sólo puede ser cumplido si se adquieren previamente los paquetes turísticos que ofrecen los operadores privados existentes, lo que dificulta la accesibilidad de los pescadores locales o de otros puntos de la provincia.

Ninguno de los tres ambientes se encuentra en un itinerario turístico, aunque desde localidades como Gobernador Gregores, se promociona el destino lago Strobel. Tanto éste último como río Capitán poseen potencial para desarrollo de otras tipologías turísticas al estar ubicados en zonas con alto valor paisajístico y en recursos arqueológicos. Situación similar ocurre en lago Olnie, que está ubicado en cercanías a la Ruta Provincial N41, la cual está siendo fuertemente fomentada como ruta turística por la Secretaria de Turismo provincial en la actualidad.

\section{e) Beneficios de la pesca deportiva}

El aprovechamiento de los recursos acuáticos e ícticos ha provocado una mayor preocupación por la conservación del ambiente. Los entrevistados han señalado que antes de cada inicio de temporada, se realizan tareas de mantenimiento en el lodge y en el entorno, y se sustentan estudios científicos para conocer el estado de los recursos pesqueros. Ello se ve reflejado en la valoración que han obtenido en estos aspectos, ya que los tres ambientes, han obtenido la mayor calificación en el estado de conservación del recurso y del entorno.

Otro de los beneficios, mencionados por los operadores privados, fue el aporte que realizan al movimiento socioeconómico provincial. Por una parte, por la generación de empleo directo (guías, quienes trabajan en el lodge y lo gerencian); por otro, en la compra de insumos para abastecer los distintos alojamientos, que fundamentalmente proceden de la localidad de Gobernador Gregores. Además, se producen ingresos por los aportes impositivos y las licencias de pesca que deben adquirir los pescadores internacionales, lo cual también genera un flujo de divisas para el Estado provincial.

\section{f) Rol del Estado en relación a la pesca deportiva}

La Secretaría de Turismo de la Provincia posee tres actividades pilares a las cuales destina mayormente sus esfuerzos; la pesca deportiva es uno de ellos, junto con el senderismo y los recursos paisajísticos. Son los encargados de organizar y gestionar todas las festividades de pesca que existen en diferentes localidades de Santa Cruz.

En conjunto con algunos agentes del sector privado, como la empresa turística "Tres Amigos Outfitters", organizan y dictan el curso para Guía de Pesca, que habilita a quienes lo aprueban a registrarse y desempeñarse como tal, de forma independiente o bajo dependencia, dentro del ámbito territorial de la provincia.

La Dirección de Pesca Continental en cambio, es un organismo de control. Posee participación activa en la confección del reglamento de pesca patagónico que se presenta antes del inicio de cada temporada (noviembre-abril). Además, es el encargado de otorgar las licencias de pesca correspondientes y de corroborar que los pescadores y los establecimientos que ofrecen servicio de pesca cumplan con el reglamento.

Ambas entidades reconocen la importancia de la actividad y especialmente, su crecimiento año tras año. Sin embargo, ambos observan a este tipo de pesca como un nicho muy específico de mercado, debido a que el público responde a un interés especial, restringido sólo a los lodges de pesca, y no estarían generando un movimiento económico de envergadura similar a lo de otras actividades turísticas y comerciales más convencionales. 


\section{DISCUSIÓN}

La pesca deportiva es una actividad diversificadora en el ámbito turístico, capaz de movilizar capitales, ingresar divisas a la región y generar empleos genuinos. Cabe destacar que el mercado hacia el que va dirigido actualmente, no representa grandes números en cantidad de turistas en comparación con las que atraen las tipologías de turismo más convencionales (Ej: Turismo de naturaleza en Parque Nacional Los Glaciares). Sin embargo, la provincia posee potencial para el desarrollo de esta actividad y la captación de nuevas inversiones para emprendimientos similares a los existentes, en función de los numerosos cuerpos de agua situados en el noroeste de la provincia de Santa Cruz, muchos de ellos con poblaciones de salmónidos estables; lo que permitiría deducir que podría incrementarse en los próximos años, con sus consecuentes beneficios.

La demanda actual es principalmente internacional, siendo Estados Unidos el país que más pescadores emite. Ello fue coincidente con lo observado en la región de Aysén (Patagonia chilena), cercana al área de estudio del presente trabajo, donde $61 \%$ de los pescadores que reciben, también son procedentes de este país (Parrado y Huaquín, 2010), evidenciando el interés que existe por estas zonas australes, en su conjunto. Esto resulta relevante si consideramos que, en el año 2016, se han estimado para EEUU unos 35.8 millones de pescadores deportivos, de los cuales alrededor del $23 \%$ viaja fuera del país para desarrollar la actividad; por lo que existe un mercado potencial muy alto para nuestra provincia, que con organización y promociones adecuadas puede captar.

Los pescadores se alojan directamente en los lodges, mediante la adquisición de paquetes que incluyen los traslados, servicio de alojamiento y alimentación, por lo que no les resulta necesario utilizar los servicios de hospedaje ni gastronómicos de las localidades aledañas. En este sentido, es al menos discutible el derrame socioeconómico que produce esta actividad en las comunidades locales. A ello se suma, la distancia existente de los ambientes de pesca entre sí y con los centros urbanos más cercanos y particularmente, el estado de los caminos que dificultan el movimiento, por lo que los turistas permanecen toda su estadía en el mismo lugar. La accesibilidad física se destaca, por tanto, como una limitante de esta actividad.

Respecto a la accesibilidad legal, es decir si hay accesos públicos o privados para llegar a los sitios de pesca, resultó otro de los criterios que obtuvo menor puntaje, tanto en uno como en otros de los ambientes acuáticos considerados. Sin embargo, no se traduce en un problema grave para la operatoria normal de la pesca, ya que los visitantes poseen libre acceso a los cuerpos de agua, por acuerdos previos de cada lodge con los propietarios de la tierra. Esta situación en cambio, se transforma en una problemática si se desea desplegar la pesca deportiva para un mercado más amplio o responder a una demanda regional o nacional, ya que la accesibilidad es mayormente privada, generando potenciales tensiones sociales.

Si se analiza la actividad desde los aspectos ambientales, siendo uno de los pilares en que se apoya el turismo sustentable, cabe señalar como positivo, la preocupación de los operadores de los lodges por conservar los recursos ícticos y el entorno que los rodea, debido a que el estado óptimo de ambos es fundamental para ofrecer una pesca de calidad, posicionarse en el sector y captar nuevos pescadores, manteniendo el desarrollo normal de la misma. En este sentido, según lo mencionado por Chávez y Osorio (2006), en esta tipología turística, la conservación del ambiente ha dejado de ser un factor limitante al desarrollo para ser un elemento clave de competitividad. La sustentabilidad del turismo requiere un crecimiento en la contribución de la actividad a la economía y la sociedad por un lado, y el uso sostenible de los recursos y el medioambiente por otro.

No obstante, es necesario aclarar que la especie objeto de la pesca y que resulta el atractivo para los pescadores deportivos en estas latitudes, no es endémica, sino que es introducida (Oncorhynchus mykiss), habiendo sido señalada como una especie invasora que puede afectar 
a la fauna autóctona. La introducción de salmónidos es un fenómeno de escala mundial que ha originado un impacto negativo en los ecosistemas dulceacuícolas. Se la considera como una de las causas principales de la extirpación y disminución en la abundancia de especies en arroyos (Buria et al., 2007). Por ejemplo, en el Parque Nacional Laguna Blanca (Neuquén), la presencia de las truchas generó múltiples efectos negativos en el ambiente, tales como el aumento de la concentración de sólidos en suspensión, la disminución de la abundancia del zooplancton e incremento del fitoplancton con el desarrollo estacional de floraciones algales, la pérdida total de la cobertura de la macrófita Myriophyllum elatinoides, la desaparición de la principal población de una rana endémica (Atelognathus patagonicus), la disminución de la abundancia de aves acuáticas y el aumento de aves carroñeras y piscívoras (Ortubay et al., 2006). La conservación de los procesos ecológicos y la biodiversidad es una premisa fundamental cuando se habla de sustentabilidad ambiental en el turismo (Chávez y Osorio, 2006). Por consiguiente, sería necesario desarrollar una línea interdisciplinaria de gestióninvestigación que involucre a la comunidad (en este caso, operadores y pescadores, poblaciones aledañas y estado) no sólo en la generación de información sino también en el proceso de manejo en sí mismo (Sanguinetti et al., 2014), para que se aproveche este recurso mediante la pesca deportiva, pero cuidando que no se extienda a otros ambientes acuáticos que hoy están sin la presencia de esta especie u otras del grupo.

Una correcta gestión y manejo de los recursos, una planificación integral y ordenada, y una colaboración entre los operadores privados y las autoridades gubernamentales de manera continua son claves para el desarrollo de una actividad que ya posee renombre internacional, y por ende es una realidad en Santa Cruz.

Este trabajo, en su fase inicial, pretende ser un puntapié inicial hacia el relevamiento, valoración y reconocimiento del potencial turístico que la actividad posee, y su posible aporte hacia un turismo sustentable.

\section{AGRADECIMIENTOS}

Agradecemos el aporte financiero provisto por la Universidad Nacional de la Patagonia Austral, a través del Proyecto PI 29A-391 para desarrollar el presente estudio. Un agradecimiento especial a quienes respondieron gentilmente a las entrevistas, tanto de instituciones públicas como privadas, en especial al Sr. Julián Escalada. Asimismo, a los responsables del lodge "Eagle Valley" por permitirnos visitar las instalaciones y compartir unos días con guías de pesca y pescadores internacionales, lo cual nos ayudó a conocer y entender mejor la actividad.

\section{BIBLIOGRAFIA}

ALMENDRAS, A. (2015). Inventario y jerarquización de atractivos y recursos naturales/culturales en el tramo Austral de la RN40 (Santa Cruz). En Torre, M.G. y otros. Libro de las XII Jornadas Nacionales de Investigación Acción en Turismo - 1a ed. - Neuquén: EDUCO - Universidad Nacional del Comahue.

BIOTT, J. M. (2012). La pesca deportiva en Río Gallegos como actividad turística sustentable y recurso estratégico para la comunidad de Río Gallegos. Manuscrito. Informe Técnico del PI 29 A391/1. Universidad Nacional de la Patagonia Austral Unidad Académica Río Gallegos, Santa Cruz, Argentina. 
BIOTT, J. M. (2016). The fly fishing nation - Una forma de promover el turismo especializado en pesca deportiva. Pp. Cap. V: 175 - 190. En: NAVARRO, V. y S. FERRARI (Comp. y Edición). 2016. "Herramientas de gestión y comunicación para el desarrollo de un turismo sustentable". Universidad Nacional de la Patagonia Austral. Río Gallegos 183 pp. http://www.repotur.gov.ar/handle/123456789/6945

BURIA, L. R. J., ALBARIÑO, V., DÍAZ VILLANUEVA, B., MODENUTTI y BALSEIRO, E. (2007). Impact of exotic rainbow trout on the benthic macroinvertebrate community from Andean Patagonian headwater streams. Fundamental and Applied Limnology Archivfür Hydrobiologie, 168:145-154.

CAJADE, E. (2009). La pesca deportiva como principal atractivo turístico: Bahía San Blas. Tesis de Grado. Facultad de Ciencias Económicas. Universidad de La Plata, Buenos Aires, Argentina.

CALDERÓN VÁZQUEZ, F. J. (2010). Sostenibilidad y Planificación: Ejes del Desarrollo Turístico Sostenible. Revista Desarrollo Local Sostenible. Vol 3, No 8. Grupo Eumed.net y Red Académica Iberoamericana Local Global.

CHÁVEZ, E. y OSORIO, J. (2006). Turismo y sustentabilidad: de la teoría a la práctica en Cuba. Cuadernos de Turismo, n²17; pp. 201-221. Universidad de Murcia, España.

FERRARI, S., ALBRIEU, C., NAVARRO, V., MAZZONI, E., ESPINOSA, S., BIOTT, J. M., GARCÍA, A. y MAZZONI, E. (2000). El Gran Libro de la Provincia de Santa Cruz. Ediciones Mileniun - Alfa Centro Literario Madrid, 1323 pp.

IBAÑEZ, R. (2011). Pesca deportiva como un atractivo turístico en México. Caracterización, estimación de su demanda futura y efecto multiplicador a otros sectores. Revista de investigación en turismo y desarrollo local. Vol 4. México.

MAZZONI, E. y FERRARI, S. (2014). Paisajes de la Patagonia austral: sistematización y cartografía para el inventario de los recursos turísticos. Resumen. Actas X Jornadas Nacionales de Geografía Física. Malargüe, Mendoza. Disponible en: https://redargentinadegeografiafisica.files.wordpress.com/2014/04/trabajo-mazzoni1.pdf

MAZZONI, E. (2017). Landscapes of Southern Patagonia, Argentina. Their scenic characteristics and their importance for geoturism: 141-180. In: Rabassa, J (Ed.). Advances in Geomorphology and Quaternary studies in Argentina. Springer Earth System Sciences.

MINCETUR-VICEMINISTERIO DE TURISMO DE PERÚ. (2006). Manual para la formulación del inventario de recursos turísticos a nivel nacional (Fase ICategorización).

http://www.mincetur.gob.pe/turismo/Producto_turistico/Manual_de_Inventario_OCT2 006.pdf

MOLINA GARCÍA, A. (2013). Diagnóstico estratégico de la actividad de Pesca-Turismo en España. Ministerio de Agricultura, Alimentación y Medio Ambiente. Secr. General de Pesca. Madrid, España. 142 pp.

NAVARRO, V., BIOTT, J. M., MAZZONI, E. y ALMENDRAS, A. (2014). Modalidades y actividades turísticas en la RN40 austral (Santa Cruz). En Torre, M.G. y otros. Libro de las XII Jornadas Nacionales de Investigación Acción en Turismo - 1a ed. Neuquén: EDUCO - Universidad Nacional del Comahue.

OEA (Organización de Estados Americanos). (1978). Metodología de inventario turístico. Washington D.C. (EEUU).

OMT (Organización Mundial del Turismo). (1995). Carta de turismo sustentable, aprobada en Conferencia Mundial sobre Turismo sostenible. Islas Canarias.

OMT (Organización Mundial del Turismo). (2008). Introducción al turismo. Madrid, España. 
ORTUBAY, S., CUSSAC, V., BATTINI, M., BARRIGA, J., AIGO, J. (2006). Is the decline of birds and amphibians in a steppe lake of northern Patagonia a consequence of limnological changes following fish introduction? Asiatic Conserv.: Mar. Freshw. Ecosystems, 16: 93-105.

OUTDOOR FOUNDATION. (2018). 2018 Special report on fishing. A partneship Project of: Recreational Boating and fishing foundation \& Outdoor Foundation. Washington DC, Estados Unidos.

PARRADO, D. N., y HUAQUÍN, M. N. (2010). Caracterización de la pesca recreativa en la Patagonia chilena. Una encuesta a turistas de larga distancia en la región de Aysén. Estudios y Perspectivas en Turismo, 19 (1), 83-104.

PASCUAL, M. A., BENTZEN, P., RIVA ROSSI, C., MACKEY, G., KINNISON, M. y WALKER, R. (2001). First Documented Case of Anadromy in a Population of Introduced Rainbow Trout in Patagonia, Argentina. Transactions of the American Fisheries Society 130: 53-67.

ROBIN, C., VALENCIA, J., SANTANDER, P. y ORREGO, R. (2015). Turismo de intereses especiales: investigación de mercado sobre las motivaciones desde la perspectiva del cliente. Universidad Técnica Federico Santa María, Valparaíso, Chile.

SANGUINETTI, J., BURIA, L., MALMIERCA, L., VALENZUELA, A. E., NÚÑEZ, C., PASTORE, H., y CHEHÉBAR, C. (2014). Manejo de especies exóticas invasoras en Patagonia, Argentina: Priorización, logros y desafíos de integración entre ciencia y gestión identificados desde la Administración de Parques Nacionales. Ecología austral, 24 (2): 183-192.

SENATUR (Servicio Nacional de Turismo). (2001). Antecedentes básicos sobre la pesca deportiva en Chile. Gobierno de Chile. Santiago, Chile.

SOTO, D., ARISMENDI, I., SANZANA, J. y BARRERA, V. (2001). Evaluación, ordenación y manejo del potencial biológico para la pesca deportiva en la región de Los Lagos. Informe Proyecto FNDR. Región de Los Lagos. 320 pp.

UICN (Unión Internacional para la Conservación de la Naturaleza). (2000). 100 de las especies exóticas invasoras más dañinas del mundo: una selección del global invasive species database. Auckland, Nueva Zelanda.

UNIVERSIDAD DE COSTA RICA; INSTITUTO DE INVESTIGACIONES EN CIENCIAS ECONOMICAS. (2010). Informe final: un análisis de la contribución económica de la pesca deportiva y comercial a la economía de Costa Rica. UCR, Costa Rica.

U. S. DEPARTMENT OF THE INTERIOR, FISH AND WILDLIFE SERVICE AND U.S. DEPARTMENT OF COMMERCE, U.S. CENSUS BUREAU. (2016). National Survey of Fishing, Hunting, and Wildlife-Associated Recreation. $144 \mathrm{pp}$.

VACCA, C., SCHINELli, D., y MIRANDA, G. (2008). Turismo en Santa Cruz. Herramientas de Gestión aplicadas al Sector. Editorial Dunken, Buenos Aires. Argentina. 


\section{ANEXO}

Tabla 1. Ficha de inventario de sitio
FICHA PARA INVENTARIO DE RECURSOS
TURÍSTICOS
Nombre del Recurso: Río Barrancoso
\begin{tabular}{|l|l|l|l|l|}
\hline Grupo: & Categoría: & Tipo: & Subtipo: & Descripción:
\end{tabular}
Ubicación Provincia: Santa Departamento: Localidad:
Geográfica: Cruz
Descripción (Datos sobresalientes que detallan las características del recurso):
Particularidades:
Distancia en km a otros centros turísticos (localidades)
Propiedad del recurso: Público Privado Otro
\begin{tabular}{l|l|l|l} 
Quien está a & Desarrollo: & Conservación: & Promoción
\end{tabular}
cargo de...
¿Se encuentra este recurso en el itinerario de algún circuito turístico?
Principales limitaciones para el uso turístico:

\begin{tabular}{|c|c|c|c|c|}
\hline $\begin{array}{l}\text { Estado actual } \\
\text { de } \\
\text { conservación } \\
\text { del recurso }\end{array}$ & $\begin{array}{l}\text { Sin alteraciones } \\
\text { aparentes (6): }\end{array}$ & $\begin{array}{l}\text { Presenta algunas } \\
\text { alteraciones }(5 / 4) \text { : }\end{array}$ & Alterado (3/2): & $\begin{array}{l}\text { Muy Alterado } \\
\text { (1): }\end{array}$ \\
\hline
\end{tabular}

(Justificación):

\begin{tabular}{ll|l|l|l} 
Estado actual & Excelente & Entorno bien conservado & Entorno & Entorno muy \\
de & conservación del & $(5 / 4):$ & $(3 / 2):$ & \\
conservación & entorno (6): & $\checkmark$ & modificado (1):
\end{tabular}

del entorno

(Justificación)

Accesibilidad Durante todo el año Algunos meses al año $\square$ Durante la semana/Fines de

Física semana $\square$ Inaccesible $\square$

(disponibilidad

temporal y

medio de

acceso)

(Descripción de la disponibilidad)

Modo de acceso: Terrestre

Acuático $\square$ Aéreo

\begin{tabular}{|l|c|c|c|c|}
\hline $\begin{array}{c}\text { Accesibilidad } \\
\text { Física y } \\
\text { señalización al } \\
\text { recurso }\end{array}$ & $\begin{array}{c}\text { Muy accesible en } \\
\text { vehículos } \\
\text { estándar, bien } \\
\text { señalizado (6): }\end{array}$ & $\begin{array}{c}\text { Accesible por } \\
\text { caminos } \\
\text { consolidados, } \\
\text { señalización } \\
\text { básica (5/4): }\end{array}$ & $\begin{array}{c}\text { Accesible sólo en } \\
\text { vehículos todo terreno, } \\
\text { escasa señalización (3/2): }\end{array}$ & $\begin{array}{c}\text { Acceso solo } \\
\text { pedestre, sin } \\
\text { señalización } \\
(1):\end{array}$ \\
\hline $\begin{array}{l}\text { (Justificación) } \\
\text { Particularidad } \\
\text { del recurso }\end{array}$ & $\begin{array}{c}\text { Atributos } \\
\text { únicos y } \\
\text { excepcionales } \\
(6):\end{array}$ & $\begin{array}{c}\text { Destacados y rasgos } \\
\text { interesantes (5/4): }\end{array}$ & $\begin{array}{c}\text { Rasgos peculiares } \\
(3 / 2):\end{array}$ & Nulos (1): \\
\hline
\end{tabular}




\begin{tabular}{|c|c|c|c|c|c|c|c|c|c|}
\hline \multicolumn{10}{|l|}{ (Justificación) } \\
\hline Vulnerabilidad & \multicolumn{3}{|c|}{$\begin{array}{l}\text { Expuesto a agentes de } \\
\text { alteración constantes y } \\
\text { amenazas (1): }\end{array}$} & \multicolumn{3}{|c|}{$\begin{array}{l}\text { Expuesto a agentes } \\
\text { de alteración } \\
\text { inmediatos, } \\
\text { constantes o } \\
\text { potenciales }(2 / 3) \text { : }\end{array}$} & \multicolumn{2}{|c|}{$\begin{array}{c}\text { Agentes de } \\
\text { alteración poco } \\
\text { trascendentes }(4 / 5) \text { : }\end{array}$} & $\begin{array}{l}\text { Sin } \\
\text { presencia } \\
\text { de } \\
\text { agentes } \\
\text { de } \\
\text { alteración } \\
\text { (6): }\end{array}$ \\
\hline \multicolumn{10}{|l|}{ (Justificación) } \\
\hline $\begin{array}{l}\text { Accesibilidad } \\
\text { legal }\end{array}$ & \multicolumn{2}{|c|}{$\begin{array}{l}\text { Público y } \\
\text { muy } \\
\text { accesible } \\
\text { (6): }\end{array}$} & \multicolumn{2}{|c|}{$\begin{array}{c}\text { Público, con } \\
\text { dificultades para } \\
\text { el acceso }(5 / 4) \text { : }\end{array}$} & $\begin{array}{r}\text { Públ } \\
\text { privad } \\
\text { restric } \\
\text { (3/ }\end{array}$ & & \multicolumn{3}{|c|}{$\begin{array}{l}\text { Privado y sin posibilidades } \\
\text { de acceso (1): }\end{array}$} \\
\hline \multicolumn{10}{|l|}{ (Justificación) } \\
\hline \multicolumn{2}{|l|}{$\begin{array}{l}\text { Servicios e } \\
\text { Instalaciones }\end{array}$} & \multicolumn{2}{|c|}{$\begin{array}{l}\text { Gran variedad de } \\
\text { servicios (6): }\end{array}$} & \multicolumn{2}{|c|}{$\begin{array}{l}\text { Existencia de } \\
\text { servicios } \\
\text { básicos }(5 / 4) \text { : }\end{array}$} & \multicolumn{2}{|c|}{$\begin{array}{l}\text { Presenta servicios } \\
\text { mínimos }(3 / 2) \text { : }\end{array}$} & & $\begin{array}{l}\text { o presenta } \\
\text { ervicios ni } \\
\text { stalaciones } \\
\text { (1): }\end{array}$ \\
\hline \multicolumn{10}{|l|}{ (Justificación) } \\
\hline \multicolumn{10}{|c|}{$\begin{array}{l}\text { Servicios o instalacior } \\
\text { Alojamiento: } \\
\text { Alimentación: } \\
\text { Esparcimiento: } \\
\text { Actividades: } \\
\text { Instalaciones: } \\
\text { Otras: }\end{array}$} \\
\hline \multicolumn{2}{|c|}{$\begin{array}{l}\text { Demanda (Tipo de } \\
\text { Visitantes) }\end{array}$} & $\begin{array}{l}\text { Locales } \\
\text { (1): }\end{array}$ & \multicolumn{2}{|c|}{$\begin{array}{l}\text { Regionales } \\
\quad(2 / 3) \text { : }\end{array}$} & \multicolumn{2}{|c|}{ Nacionales (4/5): } & \multicolumn{3}{|c|}{ Internacionales (6): } \\
\hline \multicolumn{10}{|l|}{ (Justificación) } \\
\hline $\begin{array}{l}\text { Explotación } \\
\text { del recurso }\end{array}$ & \multicolumn{2}{|c|}{$\begin{array}{c}\text { Muy } \\
\text { explotado y } \\
\text { desarrollado } \\
\text { (6): }\end{array}$} & \multicolumn{2}{|c|}{$\begin{array}{c}\text { Explotado, } \\
\text { desarrollado } \\
\text { recientemente } \\
(5 / 4):\end{array}$} & \multicolumn{2}{|c|}{$\begin{array}{l}\text { Poco explotado } \\
\text { actualmente } \\
(3 / 2):\end{array}$} & \multicolumn{3}{|c|}{ Sin explotación actual (1): } \\
\hline \multicolumn{10}{|l|}{ (Justificación) } \\
\hline \multicolumn{10}{|c|}{ Valoración total: (Suma de los puntajes) : } \\
\hline \multicolumn{10}{|l|}{ Fotografías: } \\
\hline \multicolumn{10}{|l|}{ FUENTES: } \\
\hline \multicolumn{2}{|c|}{$\begin{array}{l}\text { Persona/s } \\
\text { encargada/s de } \\
\text { completar la ficha: }\end{array}$} & \multicolumn{3}{|c|}{ Institución responsable: } & \multicolumn{5}{|l|}{ Fecha: } \\
\hline
\end{tabular}


Tabla 2. Ficha de Jerarquización.

\begin{tabular}{|c|c|c|c|}
\hline \multicolumn{2}{|l|}{ Ficha de Jerarquización } & \multicolumn{2}{|c|}{$\mathbf{N}^{\mathbf{o}}$} \\
\hline \multicolumn{4}{|l|}{ Nombre del Recurso } \\
\hline \multicolumn{2}{|l|}{ Provincia/Estado: } & \multicolumn{2}{|l|}{ Categoría: } \\
\hline \multicolumn{2}{|l|}{ Departamento/Partido: } & \multicolumn{2}{|l|}{ Tipo: } \\
\hline \multicolumn{2}{|l|}{ Localidad: } & \multicolumn{2}{|l|}{ Subtipo: } \\
\hline Criterio & Valor asignado & Ponderación & Subtotal \\
\hline $\begin{array}{l}\text { Estado actual de } \\
\text { conservación del } \\
\text { recurso }\end{array}$ & & 1,5 & \\
\hline $\begin{array}{l}\text { Estado actual de } \\
\text { conservación del } \\
\text { entorno }\end{array}$ & & 1 & \\
\hline $\begin{array}{l}\text { Accesibilidad Física } \\
\text { (disponibilidad } \\
\text { temporal y medio de } \\
\text { acceso) }\end{array}$ & & 1 & \\
\hline $\begin{array}{l}\text { Accesibilidad Física y } \\
\text { señalización }\end{array}$ & & 1 & \\
\hline $\begin{array}{l}\text { Particularidad del } \\
\text { recurso }\end{array}$ & & 3 & \\
\hline Vulnerabilidad & & 1,5 & \\
\hline Accesibilidad legal & & 1 & \\
\hline $\begin{array}{l}\text { Servicios e } \\
\text { Instalaciones }\end{array}$ & & 1 & \\
\hline $\begin{array}{l}\text { Demanda (Tipo de } \\
\text { Visitantes) }\end{array}$ & & 1 & \\
\hline $\begin{array}{l}\text { Explotación del } \\
\text { recurso }\end{array}$ & & 1 & \\
\hline & & TOTAL & \\
\hline
\end{tabular}

